\title{
EDITORIAL
}

\section{LA COMPLICIDAD ENTRE LA SALUD PÚBLICA Y LA ASISTENCIA SANITARIA EN LA REDUCCIÓN DE LA MORTALIDAD CARDIOVASCULAR}

\author{
Roberto Elosua $(1,2)$ \\ (1) Grupo de Epidemiología y Genética Cardiovascular (EGEG-ULEC). Instituto Municipal de Investigación \\ Médica, Barcelona. \\ (2) CIBER Epidemiología y Salud Pública.
}

En este número de la Revista se presentan dos artículos que analizan diferentes aspectos relacionados con la mortalidad cardiovascular. Ruiz-Ramos y colaboradores analizan la tendencia de la mortalidad por enfermedades cardiovasculares en Andalucía en el periodo $1975-2004^{1}$ y Gómez de la Cámara y colaboradores analizan los factores de riesgo asociados a la mortalidad en el seguimiento durante 13 años de una cohorte de población general estudio DRECE) ${ }^{2}$. En este editorial reflexionaremos sobre algunos aspectos de la mortalidad cardiovascular centrándonos especialmente en la mortalidad por enfermedad isquémica del corazón (EIC).

En 1978 se celebró en Bethesda una conferencia para analizar los cambios en las tendencias de mortalidad por enfermedades isquémicas del corazón (EIC) ${ }^{3}$. Esta conferencia dio origen a 2 importantes estudios de base poblacional, el estudio MONICAOMS (Monitoring trends and determinants in cardiovascular disease $)^{4}$, y el estudio ARIC (Atherosclerosis Risk in Communities $)^{5}$. Éstos se diseñaron para evaluar si la

Correspondencia:

Roberto Elosua

Instituto Municipal de Investigación Médica

Dr Aiguader 88

08003 Barcelona

Correo electrónico: Relosua@IMIM.ES mortalidad por EIC estaba realmente disminuyendo y, si era así, cuál era la contribución de la disminución en la mortalidad tras un episodio agudo (letalidad) y cuál la del descenso en el número de casos (incidencia). La disminución en la letalidad está fundamentalmente relacionado con una mejora en el tratamiento de la enfermedad que se explica por el desarrollo y la utilización de fármacos de mayor eficacia y también por una mejora en el acceso a los servicios sanitarios. El descenso en la incidencia está relacionado con la prevención de este tipo de enfermedades.

El estudio MONICA-OMS y el ARIC confirmaron la disminución de las tasas de mortalidad por EIC, aunque las causas de este descenso no fueron las mismas. En el estudio ARIC $^{5}$ se observó una disminución de las tasas mortalidad por EIC explicado fundamentalmente por un descenso de la letalidad, permaneciendo estable la incidencia entre 1987 y 1994 en las 4 poblaciones americanas estudiadas. En las poblaciones del estudio MONICA el $65 \%$ de esta disminución se atribuyó a un descenso en el número de nuevos casos, mientras que el porcentaje atribuible a una menor letalidad del IAM fue menor $(35 \%)^{6}$. Datos más recientes también indican que existen diferencias entre países en la proporción del descenso de mortalidad cardiovascular atri- 
buible a mejoras en el tratamiento o en el control de los factores de riesgo ${ }^{7}$, así en Estados Unidos en el periodo 1980-2000 el $47 \%$ del descenso de la mortalidad por EIC se atribuyó a mejoras en el tratamiento de la EIC y el $44 \%$ a mejoras en el control de los factores de riesgo, mientras que en Finlandia únicamente el $23 \%$ del descenso de la mortalidad se atribuyó a mejoras en el tratamiento y el $53 \%$ a mejoras en el control de los factores de riesgo. Estas diferencias entre países pueden ser debidas a las diferentes políticas de salud pública implementadas. Por ejemplo en una región de Finlandia se puso en marcha en 1972 un programa (North Karelia Project) para reducir la incidencia de EIC consiguiendo reducir la mortalidad por EIC en un $73 \%$ en el periodo 1969 a $1995^{8}$. Por este motivo en algunos países que han implementado estos grandes programas a nivel comunitario el papel del control de los factores de riesgo y la reducción en la incidencia puede ser mayor que en otros.

Los datos que presenta Ruiz-Ramos y cols indican que aunque la mortalidad por EIC estandarizada por edad en el periodo 1975-2004 está disminuyendo, el número absoluto de muertes está aumentando. Este dato refleja una de las "ventajas" de las sociedades industrializadas: el envejecimiento de la población. Este envejecimiento se está traduciendo en un aumento de la prevalencia de las patologías crónicas y un aumento de la demanda asistencial que el sistema sanitario tiene y tendrá que afrontar.

El descenso de la mortalidad por EIC se observa tanto en hombres como en mujeres, especialmente entre los años 1998-2004 ( $\sin$ que se encuentren cambios entre finales de los años 80 y 1998). Estos datos son concordantes con los de los registros poblacionales de infarto agudo de miocardio (IAM) realizados en Cataluña: el MONICA-Cataluña y el REGICOR. En el estudio MONICA-Cataluña se observó un ligero aumento en la incidencia de infarto agudo de miocar- dio (IAM) en los hombres de 35-74 años con un ligero descenso de la letalidad del IAM $^{9}$ en el periodo 1985-1997. En el estudio REGICOR se observó un descenso estadísticamente significativo de la incidencia de IAM en los hombres de 35-64 años sin cambios significativos, aunque con una tendencia al aumento, en los hombres de 65 a 74 años, y con un descenso de la letalidad intrahospitalaria del IAM ${ }^{10}$. El conjunto de estos datos sugiere que el descenso de la mortalidad por EIC está siendo más importante en el periodo 1998-2004 y probablemente relacionado con un descenso en la incidencia de IAM o al menos un retraso en la edad de aparición, y con un descenso de la letalidad. El descenso en la letalidad está claramente relacionado con la utilización de fármacos más eficaces ${ }^{11,12}$ y probablemente con mejoras en la calidad asistencial (desarrollo de protocolos, mejora en el acceso al sistema sanitario).

La caída en la incidencia, o el retraso en la aparición del IAM, está relacionado fundamentalmente con mejoras en la prevención primaria de la enfermedad. Datos recientes de la población de Girona ${ }^{13}$ indican que en el periodo 1995-2005 la distribución poblacional del colesterol total y colesterol-LDL se ha desplazado hacia la izquierda, alcanzando uno de los objetivos de Geoffrey Rose para la disminución de la incidencia de EIC en la población ${ }^{14}$. También han mejorado el conocimiento, tratamiento y control de la hipertensión, habiendo pasado de la regla del 1/3 (1 de cada tres hipertensos conocen que son hipertensos, de éstos únicamente $1 / 3$ se tratan y de éstos sólo 1/3 tiene cifras controladas) a la del ?, aunque todavía tenemos camino por recorrer y el vaso lo podemos ver medio lleno o medio vacio. Estas mejoras en el control de la tensión arterial pueden explicar parte importante del descenso en la mortalidad por enfermedad cerebrovascular observada en Andalucía donde, además, no sólo se observa en las tasas ajustadas por edad sino también en números absolutos. 
En los últimos años hemos progresado mucho en el diagnóstico, conocimiento, tratamiento y control de los factores de riesgo y la Atención Primaria ha tenido un papel protagonista principal que hay que remarcar. Muchos médicos de Atención Primaria utilizan las estimaciones de riesgo cardiovascular o coronario como un elemento importante en la decisión de instaurar tratamiento médico para la prevención de las enfermedades cardiovasculares en general y la EIC en particular. Existe actualmente un debate sobre cuál es la función de riesgo más adecuada para su utilización en España: Framingham, SCORE, FraminghamREGICOR,... Estudios de cohorte como el DRECE, y otras colaboraciones entre diferentes grupos que están en marcha pueden servir para aportar datos adicionales a los ya existentes sobre la validez y la comparabilidad de estas funciones en nuestra población.

A pesar del descenso que se está produciendo en los últimos años en la mortalidad cardiovascular, este grupo de enfermedades continúa siendo la primera causa de mortalidad en España. Es necesario potenciar la complicidad entre la salud pública y la asistencia sanitaria a nivel de atención primaria y especializada para continuar reduciendo estas cifras y mejorar la salud de la población.

\section{BIBLIOGRAFÍA}

1. Tendencias de la mortalidad por enfermedades cardiovasculares en Andalucía entre 1975 y 2004. M Ruiz-Ramos, T Hermosín Bono y F Gamboa Antiñolo. Rev Esp Salud Pública. 2008; 82:395-404.

2. Seguimiento de 1991 a 2004 de la mortalidad y los factores de riesgo emergentes en una cohorte de población general española. Estudio DRECE III (Dieta y riesgo de enfermedades cardiovasculares en España). A Gómez de la Cámara, MA Rubio Herrera, JA Gutiérrez Fuentes, JA Gómez Gerique, J del Campo, C Jurado Valenzuela, J Lapetra Peralta, E Menús Palazón y N Rodríguez Pascual. Rev Esp Salud Pública. 2008; 82:415-22.
3. Havlik RJ, Feinleib M, eds. Proceedings of the Conference on the Decline in Coronary Heart Disease Mortality, October 24-25, 1978. Washington DC: National Heart, Lung and Blood Institutye, US Department of Health, Education, and Welfare; 1979. NIH publication $\mathrm{n}^{\circ} 79-1610$.

4. WHO MONICA Project Principal Investigators. The World Health Organisation MONICA project (monitoring trends and determinants in cardiovascular disease): a major international collaboration. J Clin Epidemiol. 1988;41:105-14.

5. Rosamond WD, Chambless LE, Folsom A, et al. Trends in the incidence of myocardial infarction and in mortality due to coronary heart disease, 1987 to 1994 . N Engl J Med. 1998;339:861-7.

6. Tunstall-Pedoe H, Kuulasma K, Mähönen M, Tolonen H, Ruokokoski E, Amouyel P, for the WHO MONICA Project. Contribution of trends in survival and coronary-event rates to changes in coronary heart disease mortality: 10-year results from 37 WHO MONICA Project populations. Lancet. 1999;353:1547-57.

7. Ford ES, Ajan UA, Croft JB, Critchley JA, Labarthe DR, Kottke TE, Giles WH, Capewell S. Explaining the decrease in U.S. deaths from coronary disease, 1980-2000. N Engl J Med. 2007;356:2388-98.

8. Puska P. The North Karelia Project: from community intervention to national activity in lowering cholesterol levels and CHD risk. Eur Heart J. 1999;1(Suppl.):S9-13.

9. Sans S, Puigdefabregas A, Paluzie G, Monterde D, Balaguer-Vintró I. Increasing trends of acute myocardial infarction in Spain: the MONICA-Catalonia Study. Eur Heart J. 2005;26:505-15.

10. Gil M, Martí H, Elosua R, Grau M, Sala J, Masiá R, Pérez G, Roset P, Bielsa O, Vila J, Marrugat J. Análisis de la tendencia en la letalidad, incidencia y mortalidad por infarto de miocardio en Girona entre 1990 y 1999. Rev Esp Cardiol. 2007;60:34956.

11. Gil M, Marrugat J, Sala J, Masiá R, Elosua R, Albert X, Pena A, Vila J, Pavesi M, Pérez G. Relationship of therapeutic improvements and 28-day case fatality in patients hospitalized with acute myocardial infarction between 1978 and 1993 in the REGICOR study, Gerona, Spain. The REGICOR Investigators. Circulation. 1999;99:1767-73.

12. Sala J, Marrugat J, Masiá R, Porta M. Improvement in survival after myocardial infarction between $1978-85$ and $1986-88$ in the REGICOR 
study. (Registre GIroní del COR) registry. Eur Heart J. 1995;16:779-84

13. Grau M, Subirana I, Elosua R, Solanas P, Ramos $\mathrm{R}$, Masiá R, Cordón F, Sala J, Juvinyà D, Cerezo C, Fitó M, Vila J, Covas MI, Marrugat J. Trends in cardiovascular risk factor prevalence (1995-20002005) in northeastern Spain. Eur J Cardiovasc Prev Rehabil. 2007;14:653-9.

14. Rose G. Sick individuals and sick populations. Int J Epidemiol. 1985;14:32-8. 